\title{
Conservative Treatment of latrogenic Ascending Aortic Hematoma Post-Rotablator Coronary Procedure
}

\author{
Maxime Doutriaux ${ }^{1}$ Benjamin Pariente ${ }^{1} \operatorname{Jad}_{Z^{2}}$ Bhnan $^{1} \quad$ Xavier Marchand $^{1}$ \\ ${ }^{1}$ Department of Cardiology, CHI Poissy-Saint-Germain-en-Laye Site \\ Hospitalier de Poissy, Poissy, France \\ Address for correspondence Maxime Doutriaux, MD, Department of \\ Cardiology, CHI Poissy-Saint-Germain-en-Laye Site Hospitalier de \\ Poissy, 10 rue du Champs Gaillard, Poissy, 78300, France \\ Thorac Cardiovasc Surg Rep 2020;9:e47-e50. \\ (e-mail: maximedoutriaux@gmail.com).
}

\begin{abstract}
Background Aortic intramural hematoma due to coronary artery dissection is a rare and serious complication during percutaneous coronary intervention.

Case Presentation A 78-year-old female patient was admitted for diagnostic coronarography in the context of stable angina. The coronarography showed an asymmetric

Keyword

- aortic disease

- imaging

- aorta

- percutaneous coronary intervention and significate calcification in the ostium of the right coronary requiring Rotablator (Boston Scientific) procedure complicated by iatrogenic ascending aortic hematoma. After surgical advice, a conservative approach was decided with total hematoma resorption and recovery 1 week later.

Conclusion With stable patient, conservative treatment may be suitable after aortic hematoma due to coronary dissection.
\end{abstract}

\section{Background}

Aortic intramural hematoma (IMH) due to coronary artery dissection is a very rare but serious complication during percutaneous coronary intervention. In the literature, the incidence of this complication is around $0.06 \%{ }^{1}$ Most of the previously reported cases showed this specific complication during angioplasty of the right ostium coronary. ${ }^{2-5}$

To our knowledge, there are no official recommendations regarding the management of this iatrogenic complication. Two strategies are suggested by vascular surgeons: surgery ${ }^{2}$ or conservative therapy with repeated computed tomography (CT) of the aorta to follow the aortic IMH evolution. ${ }^{3,4}$ In case of hematoma extension during CT scan follow-up or clinical instability, a surgical intervention is preferable.

After bibliographic research, none of the previously reported cases occurred after using a coronary Rotablator (Boston Scientific) device in the right ostium coronary.

This case reports an acute and iatrogenic ascending aortic hematoma secondary to an ostial right coronary Rotablator procedure. After clinical and scannographic evaluations, a conservative strategy was initially chosen to manage our patient.

received

April 29, 2020

accepted after revision

May 26, 2020

\section{Case Presentation}

A 78-year-old Middle Eastern female patient was admitted to our hospital in early 2020 for diagnostic coronarography in the context of stable angina for several weeks.

Her medical history showed type 2 diabetes (insulindependent), hypertension, hyperlipidemia, paroxystic atrial fibrillation (anticoagulated by a direct factor Xa inhibitors: rivaroxaban $20 \mathrm{mg} /$ day), moderated overweight with a body mass index at $28 \mathrm{~kg} / \mathrm{m}^{2}$, and an ischemic cardiopathy (with three revascularization procedures since 2008 on the left coronary artery).

The last oral administration of rivaroxaban was in the evening, the day before intervention. Preinterventional hemostatic assessment on the morning of intervention (8 hours before intervention) was normal (activated cephalin time ratio $=1.11$; prothrombin time $=111 \%$; blood platelet $=327 \mathrm{G} / \mathrm{L}$ ).

Electrocardiogram showed a normal sinus rhythm without any repolarization disorder. The transthoracic echocardiogram (TTE) showed inferior hypokinetic trouble with moderate alteration of left ventricular ejection fraction (45\%).

The diagnostic coronarography showed an asymmetric and significate coronary calcification of the ostium and the
License terms Stuttgart · New York
DOI https://doi.org/

10.1055/s-0040-1715431. ISSN 2194-7635.

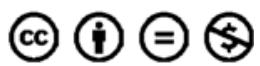




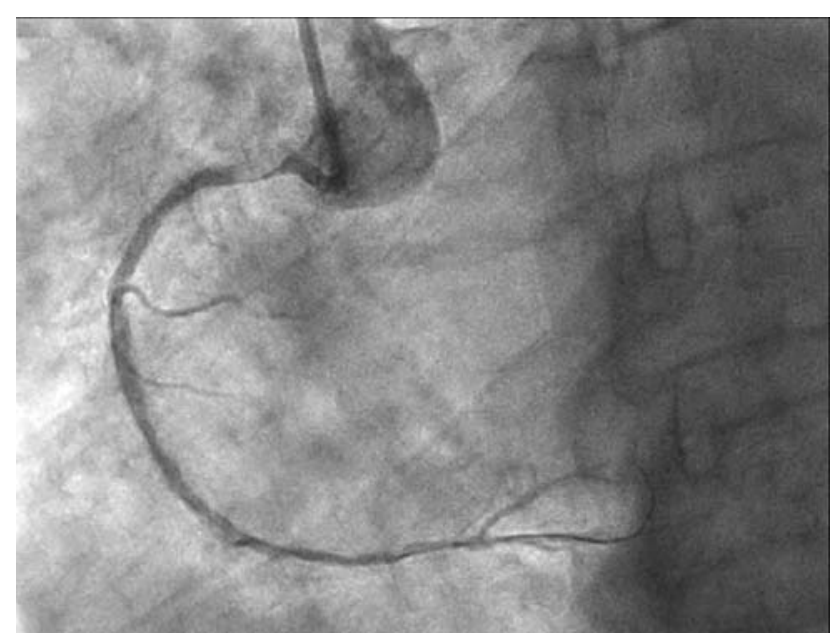

Fig. 1 Diagnostic coronarography with calcification of ostio-proximal right coronary.

initial part of the right coronary ( - Fig. 1). After predilatation failed with compliant and then noncompliant balloons, we stopped the procedure and discussed the case during a multidisciplinary meeting. A rotational atherectomy using Rotablator was decided.

One week later, we used a Rotablator device with burr sizes of $1.25 \mathrm{~mm}$ in the ostial and initial part of the right coronary. After two runs, and without any clinical symptoms, the injection control revealed an extravasation of the iodinated contrast to the ascending aorta (-Fig. 2), which was related to ostial right coronary dissection. Thinking that the aortic hematoma was driven by the ostial right coronary dissection with retrograde extension to the aortic wall, we immediately decided to implant a drug-delivery stent (ORSIRO $3 \times 13 \mathrm{~mm}$, Biotronik) in the ostium and the proximal segment of the right artery to seal the artery dissection (-Fig. 3) and stabilize the aortic IMH.

A CT scan of the ascendant aorta was performed directly after the procedure ( - Fig. 4) and found no aortic dissection

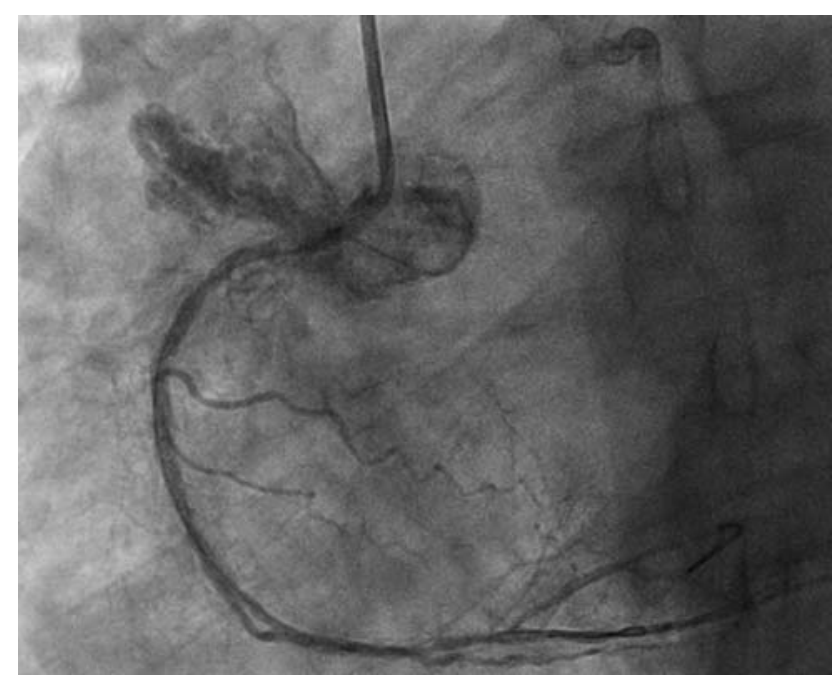

Fig. 2 Contrast extravasation from ostial right coronary to ascending aorta after Rotablator device.

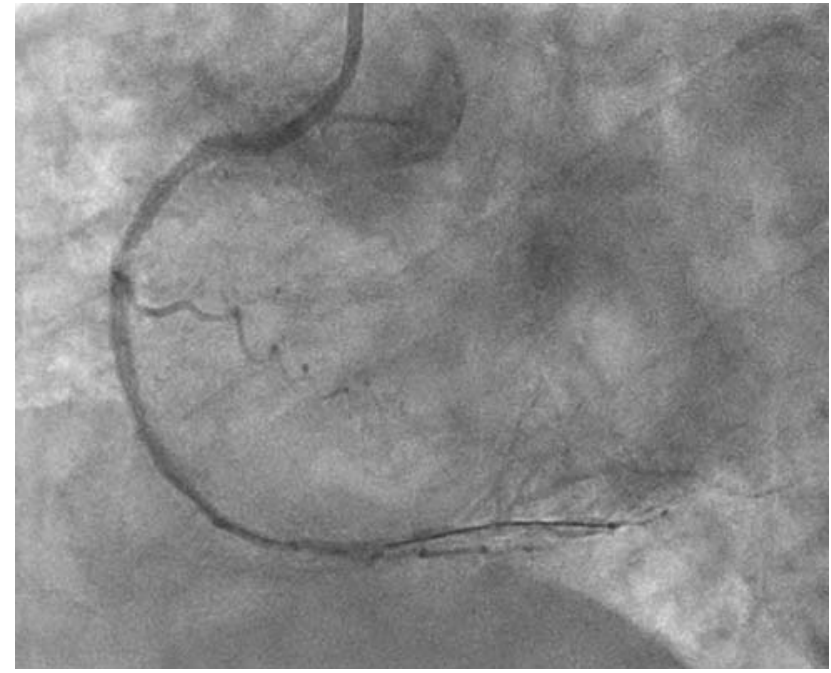

Fig. 3 Ostial right coronary after stent implantation.

(AD) but an aortic IMH, going from the ostial right coronary (with contrast product extravasation) to the arch of the aorta ( $5 \mathrm{~mm}$ thick). The TTE did not show any valvular aortic regurgitation. After surgical advice, a conservative approach was decided and the patient was transferred to the cardiologic intensive care unit for medical monitoring.

We decided to stop the anticoagulation (by direct factor Xa inhibitors) until hematoma resorption (checked by iterative CT scan on day 2 and day 7) and a blood pressure monitoring for maximal systolic pressure of less than $140 \mathrm{~mm} \mathrm{Hg}$. Follow-up consisted of repeated CT scan on day 2 and 1 week.

The first CT scan control on day 2 showed a stable hematic collection (5 $\mathrm{mm}$ thick), without any hematoma resorption or dilatation of the ascending aorta.

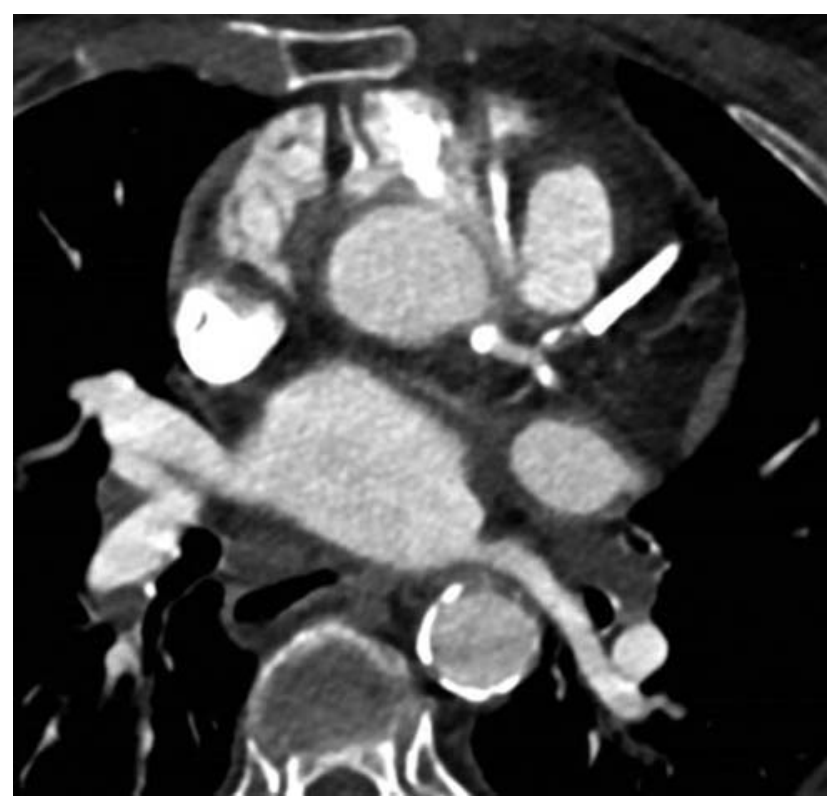

Fig. 4 Initial no injected CT-scan: contrast extravasation from ostial right coronary to ascending aorta. 


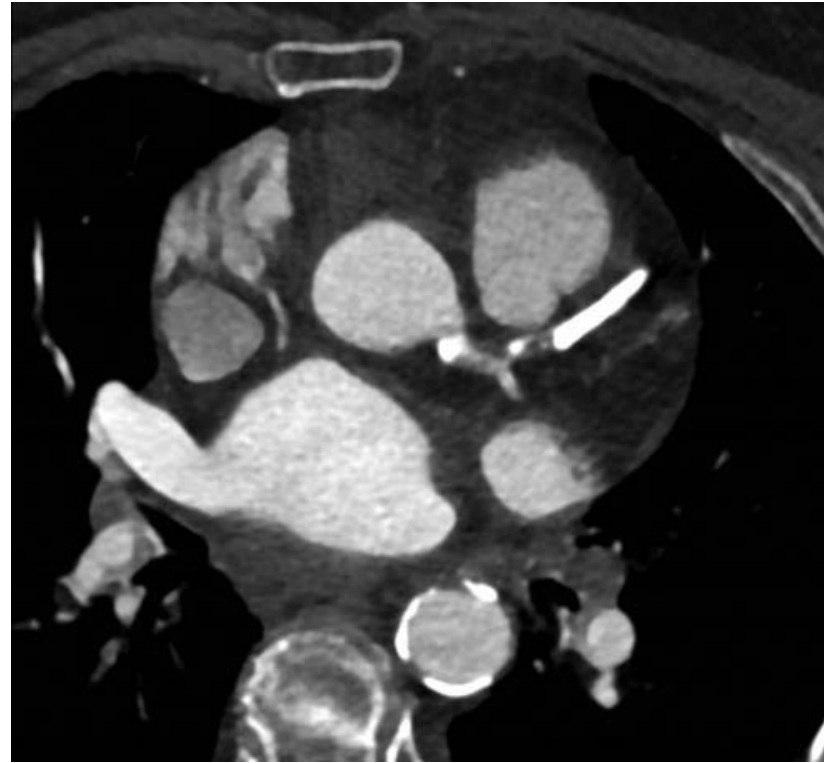

Fig. 5 Day 7 with injected CT-scan: aortic hematoma resorption without contrast extravasation.

The second CT scan control on day 7 (-Fig. 5) showed total resorption of aortic hematoma. No clinical or biological complications appeared during the monitoring period of 10 days, authorizing reintroduction of the oral anticoagulation, in association with the double antiplatelet therapy (including Lysinacetylsalicylat (100 mg/300 mg) and clopidogrel). After collegial discussions and according to recent official recommendations, ${ }^{6}$ we decided to maintain tri-therapy for 1 month and then rivaroxaban and Lysinacetylsalicylat $(100 \mathrm{mg} /$ $300 \mathrm{mg}$ ) for 1 year followed by rivaroxaban alone thereafter.

The patient left the hospital without any chest symptoms after spending 10 days in the cardiologic intensive care unit.

\section{Discussion}

IMH and AD have similar prognosis and therapeutic implications. ${ }^{7}$ The Stanford classification, commonly used in AD, may be useful for classification and therapeutic strategy in aortic hematoma. In our case, with aorta dissection limited to its ascending portion, a surgical intervention should be performed, but a conservative approach has been used in several cases with excellent long-term results. ${ }^{1,8}$ After collegial discussions with thoracic and vascular surgeons, we decided to treat our patient with the conservative strategy.

The literature shows that iatrogenic acute aortic syndrome (including IMH and AD) from coronary dissection has a lower mortality rate than spontaneous one, as well as fewer complications. ${ }^{9}$

It may be partially explained by immediate treatment, with stenting implantation at the site of the coronary dissection, which limited the extension of the aortic lesion. Plus, spontaneous aortic lesions are usually associated with significant degeneration of the media, facilitating the propagation of the dissection.

Most other reported cases have involved the right coronary artery. Some explanations in literature suggest that the larger ostium of the left main coronary artery may decrease the incidence of catheter-induced trauma. We also know that extensive coronary disease and vessel calcification, like in our case, increase the risk of complications such as coronary dissection. These procedures sometimes require aggressive manipulation of catheters or use of specific devices, such as Rotablator, ${ }^{10}$ which are more aggressive than in classical angioplasty procedure.

Nevertheless, no previous case reports described ascending aorta hematoma related to the ostial right coronary postRotablator procedure. The fact that Rotablator devices are not currently used in daily practice but reserved for few specific indications may explain the lack of similar cases.

\section{Conclusion}

In case of iatrogenic aortic hematoma due to coronary dissection, a multidisciplinary management with surgical advice is recommended to choose optimal therapeutic strategy. When patients are clinically and hemodynamically stable, without any chest pain or aortic valvular regurgitation on the TTE, a conservative strategy may be proposed.

In case of a conservative strategy, as used here, follow-up with repeated CT-scan and TTE (to eliminate aortic valvular regurgitation) resulted in aortic recovery and allowed discharge of the patient after total resorption of hematoma.

In conclusion, our case confirmed that a conservative approach in ascending aortic hematoma due to coronary artery dissection seems to be a viable alternative to an invasive surgery in a stable patient, even after a Rotablator procedure.

Conflict of Interest

None.

\section{References}

1 Núñez-Gil IJ, Bautista D, Cerrato E, et al; Registry on Aortic Iatrogenic Dissection (RAID) Investigators. Incidence, management, and immediate- and long-term outcomes after iatrogenic aortic dissection during diagnostic or interventional coronary procedures. Circulation 2015;131(24):2114-2119

2 Welch TD, Foley T, Barsness GW, et al. Iatrogenic aortic dissection ... or intramural hematoma? Circulation 2012;125(09):e415-e418

3 Muhyieddeen K, Samim A, Roberts M, Srikanth S. A case of iatrogenic aortic intramural hematoma. Methodist Debakey Cardiovasc J 2017;13(01):37-38

4 de Barros e Silva PG, Aquino T, Resende MV, et al. Resolution of an acute aortic syndrome with aortic valve insufficiency post-PCI. Am J Case Rep 2014;15:508-513

5 Shorrock D, Michael TT, Patel V, et al. Frequency and outcomes of aortocoronary dissection during percutaneous coronary intervention of chronic total occlusions: a case series and systematic review of the literature. Catheter Cardiovasc Interv 2014;84(04): 670-675

6 Valgimigli M, Bueno H, Byrne RA, et al; ESC Scientific Document Group; ESC Committee for Practice Guidelines (CPG); ESC National Cardiac Societies. 2017 ESC focused update on dual antiplatelet therapy in coronary artery disease developed in collaboration with EACTS: the Task Force for dual antiplatelet therapy in coronary artery disease of the European Society of Cardiology (ESC) and of the European Association for Cardio-Thoracic Surgery (EACTS). Eur Heart J 2018;39(03):213-260 
7 Harris KM, Braverman AC, Eagle KA, et al. Abstract 17170: Acute aortic intramural hematoma: an analysis from the International Registry of Acute Aortic Dissection. Circulation 2012;126(11, Suppl 1):S91-S96

8 Roumy A, Kirsch M, Prêtre R, Niclauss L. Spontaneous regression of a large iatrogenic dissection of the ascending aorta. Aorta (Stamford) 2016;4(06):226-228
9 Rylski B, Hoffmann I, Beyersdorf F, et al. Iatrogenic acute aortic dissection type A: insight from the German Registry for Acute Aortic Dissection Type A (GERAADA). Eur J Cardiothorac Surg 2013;44(02):353-359, discussion 359

10 Iannopollo G, Gallo F, Mangieri A, et al. Tips and tricks for rotational atherectomy. J Invasive Cardiol 2019;31(12):E376-E383 